\title{
Application des modèles aux études de ruissellement urbain : le programme RERAM
}

\author{
Use of models for urban runoff studies : \\ the RERAM program
}

\author{
Michel Desbordes \\ Laboratoire d'Hydrologie Mathématique \\ Université des Sciences et Techniques du Languedoc, Montpellier
}

\section{Introduction}

Depuis une dizaine d'années, de nombreux programmes de recherche en hydrologie urbaine sont en cours [1]. Ils ont pour but une meilleure connaissance des conséquences sur le cycle de l'eau de l'urbanisation et des activités humaines qui s'y rattachent [2]. Les premiers résultats de ces programmes ont trouvé des applications immédiates dans le domaine de l'assainissement urbain et principalement celui de l'évacuation des eaux pluviales. Il s'agit en particulier de la modélisation généralisée des phénomènes hydrologiques intervenant dans la transformation de la pluie en ruissellement et écoulement, sur un bassin urbain et dans son réseau de drainage.

Après une période d'élaboration de prototypes, on assiste aujourd'hui à l'apparition d'un nombre croissant de modèles d'assainissement, couvrant une gamme assez variée des problèmes rencontrés dans ce domaine. Il ne s'agit pas d'une nouvelle mode, mais simplement d'une volonté, de plus en plus fréquemment manifestée, d'analyser le fonctionnement d'ouvrages coûteux et de s'assurer de leur efficacité. Le programme RERAM, mis au point à la demande de l'Administration française, appartient à cette génération d'outils nouveaux. C'est un moyen d'analyse très puissant. Cependant, sa conception et sa mise en œuvre ont été étudiées pour faciliter son emploi par des techniciens de l'assainissement, sans que ces derniers soient obligés de devenir des spécialistes de l'hydrologie urbaine et de l'informatique.

La modélisation en assainissement : une nécessité

La situation de l'assainissement en France est comparable à celle rencontrée dans les autres pays industrialisés [1]. Elle est la conséquence d'une très forte croissance des agglomérations, marquée en particulier par une imperméabilisation sans cesse accrue des sols, au cours des trente dernières années. Elle est également la conséquence du manque de souplesse des équipements classiques d'assainissement, mais aussi de l'intérêt très relatif qui leur a été accordé par rapport à d'autres équipements d'infrastructure urbaine. Indépendamment du fait qu'il y a eu dans ce domaine un retard considérable, qui a contribué à tempérer les études prospectives, les méthodes et outils en usage jusqu'à ce jour ne pouvaient encourager de telles études. Au fil des ans, souvent pour faire face à un problème ponctuel dont la résolution semblait urgente, on a enfoui dans le sous-sol des villes des ouvrages toujours plus nombreux, dont la durée de vie s'est révélée être souvent supérieure à celle de leur vocation initiale. Les conséquences sont aujourd hui connues: certaines grandes agglomérations sont munies de réseaux inextricables, labyrinthes au fonctionnement confus, complétés d'année en année par de nouveaux dédales, manifestant leur existence souterraine par des désordres en surface de plus en plus fréquents. La croissance des villes et l'imperméabilisation des sols conduit à l'observation de véritables crues urbaines sous l'effet d'orages qui ne sont pas nécessairement de nature exceptionnelle, au point qu'elles peuvent localement mettre des vies humaines en danger. Le fonctionnement anormal des déversoirs d'orage de réseaux surchargés, les branchements erronés ou clandestins, l'inefficacité de certains ouvrages de traitement, menacent la qualité des milieux récepteurs déjà gravement atteints. La pollution de ruissellement pluvial, conséquence de nos modes de développement, atteint des niveaux inquiétants dans les grandes agglomérations [3]. Les efforts de la collectivité, pour combattre ces nuissances, conduisent à des investissements de plus en plus coûteux, atteignant des sommes comparables à celles consacrées à la voirie [4]. Aujourd'hui, l'assainissement est de moins en moins considéré 
comme une conséquence banale du développement urbain, et peut même, dans certains cas, en devenir une limite.

Cependant les méthodes d'analyse et les outils de calcul en usage jusqu'à ce jour, et qui relèvent de l'ancienne conception de l'assainissement, ne permettent pas une résolution des problèmes actuels [5]. L'étude des phénomènes hydrologiques et hydrauliques en milieu urbain montrent leur complexité et leur grande variabilité dans l'espace et le temps. Cette dernière ne peut être reproduite au moyen des formules classiques, trop globales, et ne rendant compte que de valeurs moyennes de ces phénomènes [6]. Or, chaque bassin urbain et les problèmes d'assainissement qui y sont associés, tendent à devenir un cas particulier, constitué par la combinaison de phénomènes hydrologiques très variables, de caractéristiques d'occupation des sols hétérogènes, et de réseaux ou d'ouvrages existants dont le fonctionnement relève souvent lui-même du cas particulier. Dans ces conditions, seule une étude fine de l'évolution dans le temps et l'espace des phénomènes hydrologiques et des modes de fonctionnement des ouvrages existants ou projetés, peut conduire à une meilleure connaissance de l'origine des problèmes et à la mise en œuvre de meilleures solutions. Cette nécessité d'une analyse spatio-temporelle conduit alors, soit à l'expérimentation, soit à la simulation des phénomènes. Cette dernière est d'ailleurs la seule envisageable en l'absence de possibilités de mesure, ou dans le cas de projets. Elle peut être physique grâce à l'emploi de modèles réduits, ou analogique ; elle peut être mathématique dans la mesure où l'on peut traduire l'évolution spatio-temporelle des phénomènes en langage mathématique. En raison de la nature fortement aléatoire des phénoménes hydrologiques, leur observation pour la mise au point de modèles purement empiriques serait longue et coûteuse. En outre, les possibilités d'extrapolation fiable de tels modèles à des bassins non jaugés nécessiteraient de longues campagnes de mesure sur des sites d'autant plus nombreux qu'il s'agit de cas particuliers. De même, les difficultés de reproduire artificiellement la structure des averses ou la variété des modes de cheminement du ruissellement sur des surfaces de toutes natures, limitent considérablement l'emploi de modèles réduits. Quant aux modèles analogiques, ils sont également difficilement concevables, en-dehors de simulateurs électroniques coûteux qui, en outre, ne sauraient être calés sans une expérimentation préalable importante.

Ainsi, seule la simulation mathématique semble réaliste. Elle doit être menée à partir d'une analyse déterministe (parfois probabiliste) des phénomènes intervenant dans la transformation de la pluie en ruissellement sur les surfaces et écoulements dans les réseaux de drainage. Elle doit être également étayée par des mesures pour vérifier la validité de certaines hypothèses ou déterminer les valeurs de certains paramètres physiques qu'elle met en jeu (intensités d'averses sur diverses durées, coefficients de rugosité, etc...).

\section{Applications des modèles en assainissement}

Les applications sont fort nombreuses, car elles ont trait à l'ensemble des problèmes rencontrés, depuis la programmation, à plus ou moins long terme, des ouvrages liés au développement probable des agglomérations jusqu'à l'entretien ou la gestion des ouvrages existants.

En matière de programmation à moyen ou long terme, les modèles devraient permettre d'analyser rapidement les conséquences sur l'assainissement de certaines stratégies de développement, qu'il s'agisse des dimensions des ouvrages principaux, des impacts sur l'environnement, des investissements, etc...

Une autre application est le calcul précis d'ouvrages projetés, le test de différentes solutions techniques et de leur souplesse d'adaptation à une évolution des problèmes. Se rattachent à cette demière application, d'autres nombreux types d'utilisation ayant trait à l'amélioration de la connaissance du fonctionnement des systèmes existants, ou à leurs possibilités dadaptation au développement (rénovations, renforcements, extensions d'ouvrages, etc...).

Enfin, la simulation précise du fonctionnement des ouvrages peut conduire à la définition de règles d'entretien ou de gestion (dont la gestion en temps réel). Elle peut également conduire à la promotion de nouvelles conceptions en matière d'assainissement, de nouvelles techniques ou ouvrages, etc...

D'un point de vue pratique, ces diverses applications des modèles ne seront que rarement totalement disjointes en raison de la durée de vie importante des ouvrages. Un même modèle pourra souvent être adapté à plusieurs applications. Aussi à l'heure actuelle peut-on définir approximativement trois grandes classes : les modèles de planification, les modeles de projet ou vérification et les modèles de gestion.

\section{Le programme RERAM : un modèle aux appli- cations potentielles variées}

Le programme RERAM (abréviation de Réseaux Ramifiés) a été élaboré pour le compte du Service Technique de la Direction Générale des Collectivités Locales (Ministère de l'Intérieur) et du Service Technique de 1'Urbanisme (Ministère de l'Environnement et du Cadre de Vie) qui en assure sa diffusion. L'actuelle version informatique a été mise au point par le Centre d'Etudes Techniques de l'Equipement d'Aix-en-Provence, sur la base de modèles prototypes élaborés par le Laboratoire d'Hydrologie Mathématique de Montpellier [7, 8, 9].

\section{Structure actuelle du programme}

Il s'agit donc d'un outil de simulation de l'évolution dans le temps et l'espace des phénomènes hydrologiques. Il est constitué par une chaine de modules ou sousprogrammes, reproduisant tour à tour les différentes phases de la transformation de la pluie sur un bassin en débit en un point donné du réseau d'écoulement. A ces sous-programmes de transformation sont associés des modules de lecture et traitement des données, d'organisation générale des calculs et de choix d'options de programmation et des modules de traitement des résultats. 


\section{Les modules pluviométriques}

Ils sont de deux types : les uns simulent l'évolution de la pluie dans le temps à l'épicentre de l'orage, les autres, l'évolution dans l'espace de la pluie.

Les premiers correspondent à 3 options d'utilisation du programme. Il s'agit d'une part de l'introduction en donnée d'une pluie observée quelconque. Cette pluie est discrétisée au pas de temps de calcul du programme. Il s'agit d'autre part d'une pluie synthétique, dite pluie de projet simplifiée, et pour laquelle peut être définie une probabilité d'occurrence ou période de retour. Cette pluie de projet, de forme doublement triangulaire, a été mise au point par le L.H.M. à partir de l'étude statistique de longues séries pluviographiques et d'une étude de sensibilité des modèles de ruissellement. De nombreuses publications ont déjà été consacrées à cette pluie de projet [7]. Il s'agit enfin de la simulation, sur une durée donnée, d'une série d'événements pluvieux de même nature que le précédent, effectuée par tirage au hasard dans les lois statistiques des variables qui les définissent et qui sont au nombre de quatre : le nombre annuel d'événements, l'intensité moyenne sur une durée de 4 heures, l'intensité moyenne sur une période de pluie intense de durée comprise entre 15 minutes et 1 heure et la position de cette période au sein de l'événement de 4 heures.

Les seconds simulent l'abattement spatial et le déplacement des orages. L'abattement spatial peut être décrit soit à partir de pluies observées simultanément en différents points d'un bassin, en utilisant une technique fondée sur la méthode des polygones de Thiessen, soit en supposant que les isohyètes sont des cercles concentriques autour de l'épicentre. La relation utilisée actuellement par le modèle, est un coefficient d'abattement $a$, fonction de la distance $r$ à l'épicentre :

$$
a=0.71 r(\mathrm{~km})^{-0.1}
$$

Cette expression dérive de la relation d'abattement communément admise en hydrologie urbaine :

$$
a=A(\mathrm{ha})^{-0.05}
$$

$A$ étant la surface concernée par l'averse. Le déplacement des averses est simulé en introduisant en donnée la trajectoire décrite par l'épicentre et la vitesse sur cette trajectoire.

\section{Le modèle de ruissellement}

Il transforme la pluie tombant sur un bassin en hydrogramme de ruissellement à l'exutoire de ce bassin et correspondant à une entrée dans le réseau des collecteurs. Il s'agit d'un modèle conceptuel dit à réservoir linéaire, qui a été testé de nombreuses fois sur des bassins expérimentaux [10]. Le modèle est défini par deux équations. La première est l'équation de stockage :

$$
S(t)=K Q(t)
$$

$S(t)$ est le stockage d'eau au temps $t$ sur le bassin et dans son réseau élémentaire de drainage; $Q(t)$ est le débit à l'exutoire ; $K$ est l'unique paramètre du modèle. La seconde est l'équation de continuité :

$$
\frac{d S}{d t}=P(t)-Q(t)
$$

$P(t)$ étant la pluie sur le bassin à l'instant $t$. Le paramètre $K$ qui s'apparente au temps de réponse du bassin peut être obtenu par calage à partir de mesures de pluie et de débit ou prédéterminé par la relation :

$$
\begin{array}{r}
K=5.07 \times A^{0.18} \times p^{-0.36} \times(1+C)^{-1.9} \times T P^{0.21} \times \\
\times L^{0.15} \times H P^{-0.07}
\end{array}
$$

dans laquelle $K$ est en minutes, $A$ est la surface du bassin en hectares, $p$ sa pente moyenne en pourcentage, $C$ le coefficient d'imperméabilisation des sols, $T P$ la durée de pluie intense en minutes, $L$ la longueur du drain principal en mètres et $H P$ la hauteur de pluie de la période intense en millimètres.

Le domaine d'utilisation de ce modèle est assez vaste : il s'est révélé plus particulièrement efficace pour des bassins dont la taille varie de quelques hectares à quelques centaines d'hectares et qui sont relativement imperméabilisés $(C>0.2)$.

\section{Les modèles hydrauliques}

Ils ont pour fonction de simuler la propagation des hydrogrammes donnés par le modèle de ruissellement dans le réseau des collecteurs existants ou projetés et leur transformation dans les points singuliers ou ouvrages de ce réseau.

La propagation à surface libre peut être réalisée par deux méthodes. La première est une translation simple à une vitesse moyenne $\bar{V}$ de déplacement pondérée par les débits de l'hydrogramme discrétisé au pas de temps de calcul du programme, soit :

$$
\bar{V}=\frac{\Sigma V i Q i}{\Sigma Q i}
$$

La seconde est également une méthode classique connue sous le nom de Muskingum modifié et décrite par J.A. Cunge [11]. Il s'agit d'une intégration particulière, en différences finies, de l'équation différentielle du schéma de Muskingum, qui, par l'introduction d'une diffusion numérique artificielle, conduit à un résultat numériquement identique à celui que donnerait la résolution d'une équation du second ordre dite d'onde de crue diffusante. Cette propagation se traduit par un amortissement des hydrogrammes. La diffusion numérique instable doit être cependant contrôlée afin d'assurer la conservation des volumes dans la transformation. Ce contrôle est réalisé en reliant les paramètres $K$ et $x$ de l'équation de Muskingum aux caractéristiques géométriques et hydrauliques des collecteurs et en déterminant des pas d'espace (longueurs des tronçons) et de temps compatibles avec les conditions de convergence et de stabilité du schéma numérique d'intégration. Rappelons que l'équation de Muskingum est une équation de stockage linéaire semblable à l'équation 3 et qui s'écrit :

$$
S(t)=K(x P(t)+(1-x) Q(t))
$$

Le choix de cette méthode par rapport à la résolution directe d'une équation d'onde de crue diffusante, 
obtenue par simplification du système classique de SaintVenant, est dicté par des considérations de rapidité de résolution informatique. L'emploi de l'une ou l'autre des méthodes peut être choisi au départ pour l'ensemble des tronçons. Il existe également une procédure optionnelle permettant le choix automatique de l'une ou l'autre des procédures pour chaque tronçon suivant les valeurs de la pente du tronçon, de sa longueur et du débit de pointe de l'hydrogramme y entrant.

Il existe également des procédures simplifiées pour la détection des mises en charge des collecteurs. Quant aux transformations réalisées dans les points ou ouvrages singuliers, elles sont réalisées à partir d'une modélisation particulière de ces points singuliers. Pour les points de jonction, le programme opère une simple sommation des hydrogrammes propagés en ces points sauf indication contraire. Pour les autres ouvrages comme des bassins de retenue, des déversoirs, des chambres à sable, des siphons, etc..., la modélisation est conduite à partir d'une équation de stockage liée à la géométrie des ouvrages et à leur mode de fonctionnement ou de contrôle, d'une équation de continuité et de une ou plusieurs équations traduisant le mode de fonctionnement ou de contrôle ou d'évacuation de l'ouvrage.

Signalons enfin que le programme comprend une procédure optionnelle permettant d'injecter en tête de chaque tronçon du réseau un hydrogramme donné (apport de bassin extérieur, apport d'un autre réseau, etc...).

\section{Autres modules}

Il s'agit en particulier d'un module de dimensionnement de collecteurs qui peut être utilisé dans le cas de projets de réseau, mais également dans le cas de vérifications d'ouvrages existants. Il s'agit également de modules de présentation des résultats : tableaux, graphiques divers. Ces résultats peuvent d'ailleurs être traités et mis en forme par un module particulier, CERAM, extrait du programme CERA, version informatisée du modèle de Caquot [12]. Le module CERAM réalise de nombreuses opérations pour la constitution de dossiers définitifs : mise en place des collecteurs, dessin du profil en long, dessin de la ligne piézométrique, avant-métré de terrassement, estimation des coûts, etc...

\section{Domaines d'utilisation de RERAM}

En raison de sa grande souplesse, de sa simplicité de mise en œuvre et de sa grande vitesse de calcul, le programme RERAM peut recevoir de nombreuses applications :

- En programmation à moyen terme, pour définir les principales caractéristiques techniques des ouvrages à prévoir ou évaluer les conséquences d'hypothèses d'urbanisation sur les réseaux existants. La précision des calculs sera alors toute relative et l'on adoptera des procédures simplifiées avec des sous-bassins versants de grande taille : pluie synthétique, translation simple, etc... Le module CERAM permettra de dégager des coûts pour des tests d'hypothèses.

- En vérification d'ouvrages existants, avec une finesse d'analyse qui dépendra de la nature du problème.
Les pluies utilisées pourront être soit des pluies de projet simplifiées, soit des pluies réelles. Les transformations d'hydrogrammes par les ouvrages spéciaux pourront être réalisées de façon précise.

- En projet, plusieurs cas sont envisageables. Pour un avant-projet sommaire, on pourra procéder comme pour la vérification d'ouvrages existants en testant plusieurs variantes d'aménagements et en établissant grâce à CERAM un profil en long de la variante retenue. Pour un avant-projet détaillé, on pourra avoir recours à la simulation généralisée des pluies pour analyser avec plus de précision les fréquences de défaillance des ouvrages et utiliser toutes les ressources de CERAM pour la constitution du dossier.

- En exploitation, les modifications très ponctuelles d'un réseau complexe, l'élaboration de consignes d'exploitation nécessitent une grande précision de calcul. Il faudra donc le plus souvent élaborer un modèle "sur mesure" calé à partir d'observations de pluie et de débit. Cependant RERAM reste une excellente base pour de tels modèles.

\section{Evolution probable de RERAM}

Le programme RERAM né de la recherche en hydrologie urbaine, a été réalisé en vue d'une utilisation aussi vaste et aussi simple que possible par des techniciens de l'aménagement. Il comporte un ensemble de modules auxquels on a volontairement donné un niveau de précision comparable, afin de disposer d'un ensemble homogène, sans complications inutiles, peu souples, réclamant un travail préparatoire long et fastidieux, et conduisant à un allongement considérable des temps de calcul pour un raffinement de précision souvent totalement illusoire. Il n'est cependant pas un outil figé et définitif. Il bénéficiera donc des recherches poursuivies en hydrologie urbaine, qu'il s'agisse de l'aspect quantitatif, mais aussi qualitatif de l'évacuation des eaux. C'est ainsi que l'on pense enrichir prochainement ses options de nouveaux modules notamment dans le domaine de la distribution spatiale des averses (abattement variable dans le temps de l'espace). Un modèle de réponse de bassins faiblement urbanisés est également en cours d'élaboration. Dans le domaine de l'hydraulique des écoulements en réseau, des modules simplifiés de simulation des mises en charge sont à l'étude, ainsi que des modèles de représentation de maillages partiels localisés. Il est probable également que RERAM puisse prochainement disposer des modèles d'évolution de la qualité des eaux pluviales. Les campagnes de mesure menées tant en France qu'à l'étranger, sur des bassins versants expérimentaux contribueront à la mise au point définitive de ces modules.

\section{Références}

[1] Mc PHERSON M.B. - Urban hydrological modeling and catchment research : international summary. A.S.C.E. Technical Memorandum $\mathrm{n}^{\circ}$ IHP 13, $48 \mathrm{p}$. (novembre 77).

[2] U.N.E.S.C.O. - Hydrological effects of urbanization. Studies and reports in hydrology $\mathrm{n}^{\circ} 18-$ The UNESCO Press Paris, 280 p. (juillet 1974).

[3] DESBORDES M., RIBSTEIN P. - Etude de la qualité du ruissellement pluvial urbain. Synthèse bibliographique. Rapport L.H.M. 45/78, 55 p. (octobre 1978). 
[4] ROUSSET J. - Voirie et équipements urbains des collectivités locales. Ministère de l'Environnement et du Cadre de Vie - S.T.U. (mai 1978).

[5] DESBORDES M. - Le calcul des réseaux d'assainissement : les méthodes anciennes, les méthodes actuelles et leurs perspectives d'évolution. T.S.M. $\mathrm{n}^{\circ} 8-9$, p. $419-424$ (septembre 1978).

[6] DESBORDES M. - Extension du modèle de Caquot aux bassins versants hétérogènes. T.S.M. $\mathrm{n}^{\circ}$ 5, p. 223-229 (mai 1976).

[7] DESBORDES M. - Modèles de simulation de propagation de l'écoulement. Journées d'Information Nationale Eau. Ministère de l'Equipement. p. 179-187 (mai 1977).

[8] S.T.U. - Le programme RERAM. Notice d'analyse. Ministère de l'Environnement et du Cadre de Vie - S.T.U. 44 p. (juillet 1979).
[9] S.T.U. - Le programme RERAM. Notice d'utilisation. Ministère de l'Environnement et du Cadre de Vie - S.T.U. 110 p. (juillet 1979).

[10] DESBORDES M. - Réflexions sur les méthodes de calcul des réseaux urbains d'assainissemet pluvial. Thèse - Université des Sciences et Techniques du Languedoc - Montpellier, 224 p. (février 1974).

[11] CUNGE J.A., CAYLA O. - Modèle de Muskingum adapté à la simulation des propagations des écoulements dans les réseaux collecteurs. Ministère de l'Equipement. Rapport SOGREAH n ${ }^{\circ}$ R 10-983, 54 p. 9mars 1971).

[12] S.T.U. - CERA. Calcul électronique des réseaux d'assainissement. Ministère de l'Environnement et du Cadre de Vie S.T.U. (juillet 1979).

\section{Discussion}

Président : M.C. BOCQUILLON

Le Président. - Nous vous remercions très vivement de votre exposé et j'ouvre la discussion.

M. BONNIN. - Le fait qu'on ne cherche pas à traiter les eaux de pluie des réseaux séparatifs se justifie par le fait que la pollution, même si elle est occasionnellement élevée, est en général plus diluée et par suite plus acceptable par les cours d'eau. Si l'on voulait traiter les crues, il faudrait surdimensionner très largement les stations d'épuration, posant des problèmes de financement souvent insolubles; mieux vaut donc alors traiter les eaux usées quotidiennes.

M. DESBORDES. - Je n'en suis pas du tout certain. L'effet de dilution est souvent évoqué comme un remède à la pollution des caux pluviales; en réalité, les flux polluants en provenance de ces dernières peuvent être considérablement supérieurs à ceux des débits de temps sec et occasionner sur le milieu récepteur un effet de choc très grave. Ceci est encore plus vrai dans le cas de réseaux mal conçus ou mal entretenus, dans lesquels des quantités très importantes de dépôts plus ou moins fermentescibles, sont occassionnellement rejetés brutalement dans le milieu récepteur.

Ce problème de la pollution des eaux pluviales est aujourd'hui bien connu des gestionnaires de bassins de retenue.

Ce sont nos conceptions en matière d'assainissement qui ne peuvent sans doute pas s'accomoder du traitement des eaux pluviales. Mais devant l'importance du phénomène, on peut à l'heure actuelle se demander s'il est tout à fait justifié de traiter les eaux de temps sec, alors que l'on rejette sans traitement des eaux pluviales tout aussi néfastes au milieu récepteur. A l'extrême, on pourrait même imaginer d'abandonner la construction des stations d'épuration, si la seule pollution des eaux pluviales suffisait à la destruction du milieu récepteur.

M. BACHOC. - La lutte contre la pollution des eaux de ruissellement n'est certainement pas synonyme de traitement dans des stations d'épuration.

Pour lutter contre une nuisance essentielle, l'effet de choc que cette pollution provoque sur le milieu récepteur, on peut agir sur les débits au bon moment, par stockage, infiltration, dérivation. Il y a aussi des moyens de traitement spécifique à mettre au point. Des recherches sont actuellement menees sur des systèmes de séparation des matières en suspension, qui ne ressemblent pas du tout à des stations d'épuration.

Il est nécessaire de maîtriser l'envoi d'eaux pluviales polluées sur des stations classiques où elles peuvent provoquer de graves désordres.

M. DESBORDES. - La gestion, et en particulier l'entretien, des réseaux et ouvrages existants peut être un moyen de faire face au problème de la pollution des eaux pluviales. Elle devrait permettre d'adapter les modes de fonctionnement des réseaux et ouvrages existants, conçus dans l'ignorance plus ou moins totale de ce problème. Cette adaptation peut se faire par le biais du contrôle des écoulements, en retardant ou en étalant les ondes de pollution par exemple. Un bon entretien est bien sûr fondamental. On est sans doute encore loin d'une semblable gestion, en raison notamment de la faiblesse relative des budgets d'entretien des réseaux.

M. MAILLARD. - Des précédents exposés, je retiens que le paramètre le plus important est le coefficient de ruissellement. Or, les valeurs indiquées par les différents conférenciers vont de 0,19 à 0,50 dans la région de Montmorillon (M. DUJARDIN) à 0,13 à 0,40 dans la région urbaine de Bordeaux (M. CHARTON), selon l'état de saturation des sols. On se préoccupe de plus en plus du drainage des eaux parasites par les réseaux de collecte. Il doit y avoir une corrélation avec le coefficient de ruissellement et l'état de saturation des sols. A-t-on fait des recherches dans ce sens?

Deuxième point : pour les exemples traités, les coefficients de Maning-Strickler utilisés varient de 50 à 70 , ce qui répond d'ailleurs à l'instruction technique du 22 juin 1977 relative à l'assainissement urbain. Pourtant certain constructeur d'importance nationale donne pour ses fabrications un coefficient de 90. N'y a-t-il pas un risque d'erreur pour les projeteurs qui, utilisant des valeurs excessives de ce coefficient, feraient une mauvaise application du programme RERAM ?

M. DESBORDES. - M. MAILLARD m'a posé plusieurs questions. Je vais d'abord répondre à la dernière qui me semble la plus importante. Les constructeurs déclarent en effet que leurs tuyaux présentent des coefficients de Strickler à pleine section atteignant et même dépassant la valeur de 90 . $\mathrm{Je}$ ne pense pas qu'il s'agisse là d'une publicité mensongère. Cette valeur peut sans doute être atteinte, voire dépassée, sur une portion rectiligne, pour un tuyau neuf, véhiculant une eau claire ou peu chargée.

Le problème est bien différent dans le cas de collecteurs ayant déjà quelques années de mise en terre, plus ou moins bien 
posés, véhiculant des eaux agressives pour les bétons, et présentant de nombreux incidents de parcours : joints, chutes, coudes, raccordements anormaux, etc... Dans ce cas, la capacité hydraulique globale du réseau est équivalente à celle que présenterait ce même réseau s'il était bien posé et bien conçu, mais avec des collecteurs ayant des coefficients de Strickler de l'ordre de 50.

Je ne pense pas que l'on puisse alors s'en prendre au constructeur de tuyaux. Il appartient plutôt au projeteur d'être conscient du problème, de le prendre en compte dans ses calculs et d'insister sur la nécessité d'une mise en place correcte des collecteurs.

Les autres questions de M. MAILLARD se rapportaient au coefficient de ruissellement. Je ne peux y répondre en quelques minutes; nous en reparlerons après la séance si vous le voulez bien.

M. GHIO. -- Lors de la visite du réseau de ru de Marivel (géré par le Laboratoire Régional de l'Ouest Parisien), j'ai été frappé par les ouvrages de confluence et les by-pass. Connait-on les pertes de charge engendrées par ces ouvrages et peut-on les réduire?
M. DESBORDES. - On peut avoir une idée assez précise de ces pertes de charge lorsque l'on fait des mesures. M. BOCQUILLON et moi-même avons étudié certains de ces by-pass du $\mathrm{Ru}$ de Marivel et nous avons constaté que les anomalies d'écoulement provenaient plus de l'existence de régimes critiques instables que de pertes de charges anormales.

Ces problèmes d'instabilité sont très fréquents dans les ouvrages hy drauliques présentant des régimes transitoires d'écoulement plus ou moins brutaux (cas général des réseaux d'assainissement) alors que ces ouvrages ont été généralement calculés à l'aide de relations fondées sur l'existence de régimes uniformes. A ce propos, il ne semble pas que l'enseignement de l'hydraulique, et en particulier des écoulements à surface libre, dans les Ecoles d'Ingénieurs, permette d'étudier de façon précise ce genre de problèmes. Un effort de formation initiale et continue devrait être entrepris dans ce domaine.

Quant aux pertes de charge, elles peuvent être également localement importantes dans certains ouvrages spéciaux (siphons, jonctions, regards, etc...) mais elles sont généralement la conséquence d'une mauvaise conception du fonctionnment hydraulique de ces ouvrages. 